\title{
Food addiction: is there a baby in the bathwater?
}

\author{
Hisham Ziauddeen, I. Sadaf Farooqi and Paul C. Fletcher
}

Our recent Perspective article considers the evidence for a food addiction model in obesity (Obesity and the brain: how convincing is the addiction model? Nature Rev. Neurosci. 13, 279-286 (2012)) $)^{1}$. We thank Avena and colleagues for their thoughtful comments on our paper (Tossing the baby out with the bath water after a brief rinse? The potential downside of dismissing food addiction based on limited data. Nature Rev. Neurosci. 20 June 2012 (doi:10.1038/nrn3212-c1)) ${ }^{2}$. Whereas we highlighted the dangers of premature acceptance and uncritical embracement of the idea of food addiction, they counsel against its premature rejection. To be clear, our paper does not contradict this sensible view. But we do argue that existing neuroscientific evidence does not support the concept as strongly or as consistently as is often asserted. Our view echoes one that is based on reviews of the behavioural and clinical evidence concerning obesity and addiction $^{3}$.

Avena and colleagues make a clear argument that food addiction is a behavioural phenotype that is relevant to a subgroup of people with obesity and particularly to individuals who have binge-eating disorder. To this important observation, we add that a necessary prelude to further describing the frequency and clinical distribution of food addiction is to establish its validity. Specifically, before we ask ourselves who is addicted to food, we need to face and respond adequately to a series of questions. Does food addiction exist? What would we consider a basic set of criteria for proof of its existence? How might such a consideration shape experimental work aimed at its validation? For example, does it share neural mechanisms with drug addiction? This was why we suggested ${ }^{1}$ that the systematic application of cognitive neuroscience will be crucial for appraising, testing and refining the food-addiction model. We agree that such approaches are not easily transportable to the clinical setting. However, we do believe that their application is necessary in determining whether the intriguing overlap between food- and drug-related behaviours in humans goes beyond the superficial. It was the inconsistency in the (admittedly limited) existing human neuroscientific evidence that motivated our cautionary perspective. We agree that this does not justify a rejection of food addiction. Indeed, it should encourage a more detailed consideration of how to explore the concept further. Such approaches, we believe, will entail careful examination of individual variability ${ }^{4}$ and more precise clinical profiling. As Avena and colleagues note, there has so far been only one neuroimaging study directly exploring individual variability in foodaddiction scores and, indeed, only two individuals in this study met the full criteria for food addiction ${ }^{5}$.

In short, we find key points of agreement with Avena and colleagues but, whereas they argue against the premature rejection of food addiction, we argue against its premature acceptance, a stance that is based on our belief that an untested assumption should not unduly influence the interpretation of data or the formulation of policy in this field ${ }^{6}$. We argue that cognitive neuroscientific means of testing the model will advance us beyond ideas based purely on clinical overlap. This enterprise is underway: indeed the commentary is authored by scientists who have already contributed enormously in this regard. But, as we all agree, so far the results, viewed as a whole, have been neither convincing nor consistent. We do not argue that the bathwater should be carelessly tipped out, rather that we must plumb the murky water and determine whether there is a baby in there.

Hisham Ziauddeen and Paul C. Fletcher are at the Department of Psychiatry, University of Cambridge, Herchel Smith Building, Addenbrooke's Hospital, Cambridge CB2 OSZ, UK; and at the Cambridgeshire and Peterborough NHS Foundation Trust, Fulbourn Hospital, Cambridge CB21 5EF, UK.

Hisham Ziauddeen and I. Sadaf Farooqi are at the University of Cambridge Metabolic Research Laboratories, Institute of Metabolic Science, Addenbrooke's Hospital, Cambridge CB2 OQQ, UK.

Correspondence to: P.C.F. e-mail:pcf22@cam.ac.uk doi: 10.1038/nrn3212-c2

1. Ziauddeen, H. H., Farooqi, I. S. ¿ Fletcher, P. C. Obesity and the brain: how convincing is the addiction model? Nature Rev. Neurosci. 13, 279-286 (2012)

2. Avena, N. M., Gearhardt, A. N., Gold, M. S., Wang, G.-J. \& Potenza, M. N. Tossing the baby out with the bathwater after a brief rinse? The potential downside of dismissing food addiction based on limited data. Nature Rev. Neurosci. 20 June 2012 (doi: 10.1038/nrn3212-c1).

3. Wilson, G. T. Eating disorders, obesity and addiction. Eur. Eat. Disord. Rev. 18, 341-351 (2010).

4. Stice, E., Spoor, S., Bohon, C. \& Small, D. M. Relation between obesity and blunted striatal response to food is moderated by TaqIA A1 allele. Science 322 , 449-452 (2008)

5. Gearhardt, A. N. et al. Neural correlates of food addiction. Arch. Gen. Psychiatry 68, 808-816 (2011).

6. Gearhardt, A. N., Grilo, C. M., DiLeone, R. J. Brownell, K. D. \& Potenza, M. N. Can food be addictive? Public health and policy implications. Addict. 106, 1208-1212 (2011).

Competing interests statement

The authors declare competing financial interests.

H.Z. is supported by a GlaxoSmithKline-Wellcome translational medicine Ph.D. studentship. P.C.F. has received fees from GlaxoSmithKline. 